\title{
Routine use of Zenit RA, a novel chemiluminescent immunoanalyzer in autoimmune disease diagnosis
}

\author{
P. Ghillani $\cdot$ L. Dufat $\cdot$ S. Himeur $\cdot$ M. Miyara $\cdot$ \\ Z. Amoura $\cdot$ L. Musset
}

Received: 26 September 2011/ Accepted: 9 March 2012/Published online: 21 March 2012

(C) Springer-Verlag 2012

\begin{abstract}
The detection of antibodies is useful to diagnose and/or to classify autoimmune diseases as connective tissue diseases and vasculitis. Zenit RA is a fully automated immunoanalyzer. The aim of this study was to compare the predictive and discriminative performance of the Zenit RA anti-cyclic citrullinated peptide (CCP), anticardiolipin (aCL) and anti- $\beta 2$ glycoprotein 1 (aB2GP1) tests to conventional ELISAs on clinically well-defined groups of patients and to daily evaluate the determination of anti-extractable nuclear antigen (ENA), anti-double stranded DNA (dsDNA), anti-myeloperoxidase (MPO) and anti-proteinase 3 (PR3) antibodies in a hospital laboratory. Reagents available on Zenit RA analyzer exhibit good diagnostic performances, regarding sensitivity, specificity, positive and negative predictive values. Global agreements between Zenit RA and conventional tests were from 90 to $98 \%$ (Kappa-values ranging 0.56-0.94): $96 \%$ for antiCCP, 90-94\% for aCL and aB2GP1, $94 \%$ for antidsDNA, $97 \%$ for anti-ENA, $98 \%$ for anti-MPO and $95 \%$ for anti-PR3 antibodies. Zenit RA analyzer is easy to rapidly detect the most common autoantibodies in
\end{abstract}

P. Ghillani - L. Dufat $\cdot$ S. Himeur - M. Miyara $\cdot$ L. Musset Department of Immunology, Groupe Hospitalier

Pitié-Salpêtrière, Assistance Publique-Hôpitaux de Paris,

Paris, France

P. Ghillani $(\bowtie)$

Laboratoire d'Immunochimie, Département d'Immunologie, Groupe Hospitalier Pitié-Salpêtrière, 83 bld de l'Hôpital,

75013 Paris, France

e-mail: pascale.ghillani-dalbin@psl.aphp.fr

\section{Z. Amoura}

Department of Internal Medicine, Groupe Hospitalier Pitié-Salpêtrière, Assistance Publique-Hôpitaux de Paris, Paris, France autoimmune diseases. This system has a potential to provide clinically useful data within a short time. Because of the flexibility of its work modalities, it is well adapted to determine antigenic specificities in daily practice.

Keywords Zenit RA chemiluminescent analyzer . Autoimmune diseases - Antiphospholipid antibodies . Anti-CCP antibodies - Antibody screening

\section{Introduction}

The detection of antibodies is useful to diagnose and/or to classify autoimmune diseases as connective tissue diseases and vasculitis [1,2]. Classical dichotomic investigations are often time-consuming and do not allow prompt response to clinicians. Currently, the laboratory has to perform safety diagnostic tests as soon as possible to reduce hospitalization time. Zenit RA is a fully automated immunoanalyzer, provided by A. Menarini Diagnostics, for auto-immunity testing [3]. The assay is based on a two-step indirect chemiluminescent immunoassay (CLIA) using antigen-coated magnetic particles as solid phase and an antibody labeled with a dimethyl acridinium ester as detection marker.

The aim of this study was (1) to compare the predictive and discriminative performance of the Zenit RA anti-cyclic citrullinated peptide (CCP), anti-cardiolipin (aCL) and anti- $\beta 2$ glycoprotein 1 (aB2GP1) tests to conventional ELISAs on clinical well-defined groups of patients, respectively, with rheumatoid arthritis (RA) or antiphospholipid syndrome (APS); (2) to daily evaluate the determination of anti-extractable nuclear antigen (ENA), anti-double stranded DNA (dsDNA), anti-myeloperoxidase (MPO) and anti-proteinase 3 (PR3) in a hospital laboratory. 
We report the results of a comparison between the results obtained using the new fully automated Zenit RA and those from our laboratory tests for the detection of various auto-antibodies and the use of such system in various work modalities as batch mode, random access and stat priority.

\section{Patients and methods}

Serum samples from 30 healthy controls, 81 patients with well-defined rheumatoid arthritis, according to classification criteria approved by American College of Rheumatology [4], 79 patients with APS defined by revised Sapporo laboratory criteria [5], 13 patients with Sjögren syndrome, 23 patients with systemic lupus erythematosus (SLE), 6 patients with hepatitis $C$ infection and 9 patients with Waldenström disease were included in the first part of the study. Simultaneously, consecutive serum samples ( $n=393$ ) sent for anti-ENA, anti-dsDNA and/or antiMPO and anti-PR3 antibodies detection were analyzed in comparison to conventional tests. Collection of patient samples was carried out according to the local ethics committee regulations and all tests were performed according to the manufacturer's instructions.

Zenit RA assay is based on a two-step indirect CLIA using antigen-coated magnetic particles as solid phase. The nature of antigens coupled in Zenit RA reagents is shown in Table 1. During the first step, the specific antibodies present in the sample bound to the solid phase and after extensive washing, a second antibody labeled with a

Table 1 Characteristics of coupled antigens in Zenit RA reagents

\begin{tabular}{ll}
\hline Analytes & Nature of antigens \\
\hline ds-DNA & PCR product \\
SSA $60+52 \mathrm{kDa}$ & Recombinant protein \\
SSB & $48 \mathrm{kDa}$ recombinant protein \\
Sm & Protein native purified mix of subunits \\
& $\mathrm{D}, \mathrm{B}, \mathrm{E}, \mathrm{F}, \mathrm{G}$ \\
RNP & Recombinant RNP 70 kDa, RNP A \\
& $34 \mathrm{kDa}$ and RNP C 25 kDa \\
Scl70 & $72 \mathrm{kDa}$ recombinant protein \\
Jo1 & $58 \mathrm{kDa}$ recombinant protein \\
Cardiolipin & Bovine cardiolipin and human \\
$\beta 2$ Glycoprotein 1 & $\beta 2$-glycoprotein 1 protein \\
MPO & Human $\beta 2$-glycoprotein 1 protein \\
PR3 & Purified protein from human neutrophils \\
CCP & Purified protein from human neutrophils \\
\hline
\end{tabular}

ZENIT RA ENA Screen: screening panel contains nine different kinds of microparticles: SSA52, SSA60, SSb, Sm, RNP70 kDa, RNP A $34 \mathrm{kDa}$, RNP C $25 \mathrm{kDa}$, Scl-70 $72 \mathrm{kDa}$, Jo-1 $58 \mathrm{kDa}$ dimethyl acridinium ester as detection marker was added. After washing, a light signal was generated by chemiluminescent reaction and was measured. The signal was proportional to the amount of antibodies bound to the solid phase. The results were then compared with those obtained with our routinely used tests.

\section{Statistical tests}

Concordance between Zenit RA and our laboratory values was assessed by calculating kappa $(\kappa)$ coefficient. Comparison of quantitative values obtained using Zenit RA or laboratory reagents was evaluated by regression analysis.

\section{Results}

Part I: determination of anti-CCP antibodies

As consensual reported, anti-CCP antibodies are sensitive and highly specific markers for RA [6], especially in early stage $[7,8]$. In this study, 170 serum samples were analyzed comprising 81 serums samples from patients with defined rheumatoid arthritis and 89 serum samples as controls (30 from healthy individuals and 59 from patients with others diseases defined above). A global concordance of $96.5 \%(164 / 170)$ was found $(\kappa=0.93)$ and as shown in Fig. 1 , a very good correlation $(r=0.91)$ was available between CLIA and ELISA. CLIA (Zenit RA) values were fivefold lower than ELISA, as also confirmed in cut off values: $5 \mathrm{U} / \mathrm{mL}$ using CLIA and $25 \mathrm{U} / \mathrm{mL}$ using ELISA. Analysis of discrepancies showed a higher sensitivity of our ELISA test. Indeed, five patients with defined RA and with low anti-CCP antibodies titers (from 33 until $60 \mathrm{U} /$ $\mathrm{mL}$ ) were not detected by Zenit RA reagent.

However, both tests showed good discrimination performances as summarized in Table 2. Then, Zenit RA anti-

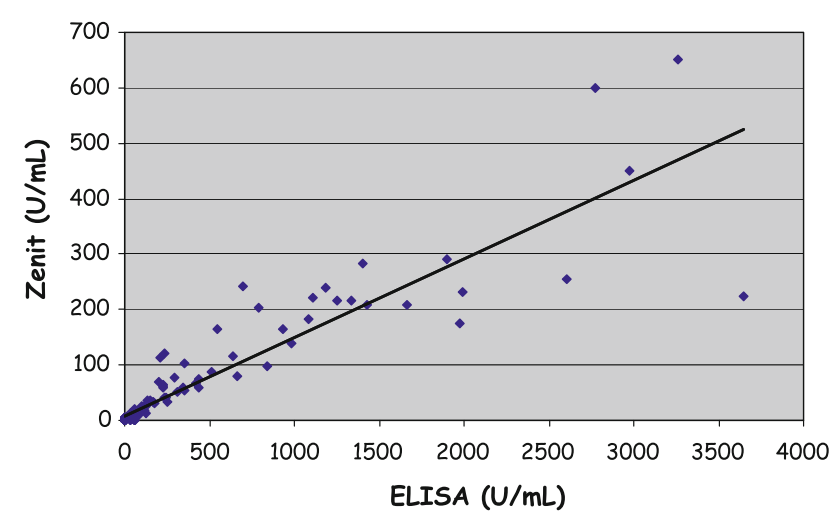

Fig. 1 Correlation between CLIA (Zenit RA) and ELISA for antiCCP antibody determination $(r=0.91)$ 
Table 2 Comparative performances of CLIA (Zenit RA) and ELISA tests for anti-CCP antibody determination

\begin{tabular}{llccc}
\hline & Sensitivity $(\%)$ & Specificity $(\%)$ & PPV $(\%)$ & NPV $(\%)$ \\
\hline CLIA (Zenit RA) & 86.4 & 100 & 100 & 89 \\
ELISA & 92.6 & 98.6 & 98.7 & 94 \\
\hline
\end{tabular}

$P P V$ positive predictive value, $N P V$ negative predictive value

Table 3 Comparative performances of CLIA (Zenit RA) and ELISA tests for APSrelated antibody determination

\begin{tabular}{lllll}
\hline & $\begin{array}{l}\text { Sensitivity } \\
(\%)\end{array}$ & $\begin{array}{l}\text { Specificity } \\
(\%)\end{array}$ & $\begin{array}{l}\text { PPV } \\
(\%)\end{array}$ & $\begin{array}{l}\text { NPV } \\
(\%)\end{array}$ \\
\hline aCL (IgG) & & & \\
CLIA & 54.4 & 97.3 & 93.5 & 75 \\
ELISA & 68.3 & 95.5 & 91.5 & 81 \\
aCL (IgM) & & & & 64 \\
CLIA & 24.1 & 95.5 & 79.2 & 63 \\
ELISA & 20.2 & 97.3 & 84.2 & 77 \\
aB2GP1 (IgG) & & & & 72 \\
CLIA & 53 & 91 & 97.2 & 63 \\
ELISA & 40 & 91 & & 65 \\
aB2GP1 (IgM) & & 95.1 & 75 & 83.3 \\
CLIA & 20.8 & 98.1 & & 78 \\
ELISA & 15.2 & & 84.8 & \\
At least one out of & & & 86.8 & \\
above antibodies & & 91.9 & & \\
CLIA & 63.3 & 91.9 & & \\
ELISA & 74.7 & & & \\
\hline
\end{tabular}

CCP assay exhibited satisfactory diagnostic value useful for clinical use regarding to the good predictive ability.

Part II: determination of APS-related antibodies

In this part, 190 serum samples were studied: 79 serum samples from patients with defined APS according to the Sapporo laboratory criteria [5, 9] and 111 controls comprising 30 serum samples from healthy individuals and 81 serum samples from patients with other diseases defined in "Patients and methods."

Four parameters were analyzed: IgG anticardiolipin (aCL IgG), IgM anticardiolipin (aCL IgM), IgG anti- $\beta 2$ glycoprotein 1 (aB2GP1 IgG) and IgM anti- $\beta 2$ glycoprotein 1 (aB2GP1 IgM), with different cut-off values in the two tests: for aCL IgG, 20 GPL U (IgG phospholipid) using Zenit and $15 \mathrm{GPL} U$ in ELISA; for aCL IgM, 10 MPL U (IgM phospholipid) using Zenit and 15 MPL U in ELISA; for aB2GP1 IgG, 20 arbitrary units (AU)/mL using Zenit and $10 \mathrm{AU} / \mathrm{mL}$ in ELISA and for aB2GP1 $\mathrm{IgM}, 10 \mathrm{AU} / \mathrm{mL}$ in either one.

Global agreements between CLIA (Zenit RA) and ELISA were from 90 to $94 \%$ [90\% for aCL IgG $(\kappa=0.75) ; 91 \%$ for aCL $\operatorname{IgM}(\kappa=0.75)$ and $94 \%$ for both aB2GP1 $\operatorname{IgG}(\kappa=0.50)$ and $\operatorname{IgM}(\kappa=0.61)]$. The comparative performances of the tests regarding sensitivity, specificity, positive and negative predictive values are shown in Table 3.

Clinical specificity was similar and high for all tests, whereas clinical sensitivity was lower in CLIA than ELISA for aCL IgG antibodies and higher for aCL IgM and aB2GP1 IgG and IgM. Taken together, the positivity of at least one out of antiphospholipid antibodies enhanced the sensitivity in respect of good specificity, NPV and PPV.

We found major discrepancies between these two tests as summarized in Table 4, in some well-defined APS patients. Discrepancies should arise from the disparities of qualitative and/or quantitative antigens, the potential alteration of epitopes structure during coating and the amount of each antigen. Indeed, Zenit RA anticardiolipin test was performed in a B2GP1-dependant manner whereas our ELISA test did not [9]. Furthermore, correlations between CLIA and ELISA values were not very good, especially for IgG isotype aB2GP1 $(r=0.63)$ and $\mathrm{aCL}$ antibodies (not calculated for aCL IgG and $r=0.78$ for aCL IgM).

These results can also reflect various panels of antiphospholipid antibodies, especially among aB2GP1 antibodies directed to various domains of B2GP1 antigen [10] and dependent on coating conditions. 
Table 4 Major discrepancies between CLIA (Zenit RA) and ELISA APS related antibody determinations in APS patients

\begin{tabular}{|c|c|c|c|c|c|c|c|c|}
\hline \multirow[t]{2}{*}{ Pathology } & \multicolumn{4}{|c|}{ CLIA (Zenit RA) } & \multicolumn{4}{|l|}{ ELISA } \\
\hline & $\begin{array}{l}\text { aCL (IgG) } \\
\text { GPL U }\end{array}$ & $\begin{array}{l}\text { aCL (IgM) } \\
\text { MPL U }\end{array}$ & $\begin{array}{l}\text { aB2GP1 (IgG) } \\
\text { AU/mL }\end{array}$ & $\begin{array}{l}\text { aB2GP1 (IgM) } \\
\text { AU/mL }\end{array}$ & $\begin{array}{l}\text { aCL (IgG) } \\
\text { GPL U }\end{array}$ & $\begin{array}{l}\text { aCL (IgM) } \\
\text { MPL U }\end{array}$ & $\begin{array}{l}\text { aB2GP1 (IgG) } \\
\text { AU/mL }\end{array}$ & $\begin{array}{l}\text { aB2GP1 (IgM) } \\
\text { AU/mL }\end{array}$ \\
\hline APS + SLE & 353 & 2 & 17 & 0 & 16 & 4 & 50 & 3 \\
\hline $\mathrm{APS}+\mathrm{SLE}$ & 808 & 2 & 570 & 2 & 392 & 86 & 360 & 4 \\
\hline $\mathrm{APS}+\mathrm{SLE}$ & 68 & 1 & 92 & 1 & 13 & 4 & 1 & 1 \\
\hline APS & 1 & 1 & 1 & 2 & 85 & 14 & 1 & 1 \\
\hline APS & 99 & 1 & 146 & 2 & 17 & 4 & 7 & 1 \\
\hline APS & 123 & 10 & 159 & 11 & 16 & 4 & 1 & 1 \\
\hline APS & 100 & 4 & 61 & 3 & 25 & 4 & 1 & 1 \\
\hline $\mathrm{APS}+\mathrm{SLE}$ & 1 & 3 & 1 & 2 & 16 & 94 & 1 & 1 \\
\hline APS & 1 & 8 & 3 & 16 & 4 & 75 & 1 & 1 \\
\hline
\end{tabular}

Positive values are mentioned in bold

APS antiphospholipid syndrome, SLE systemic lupus erythematosus

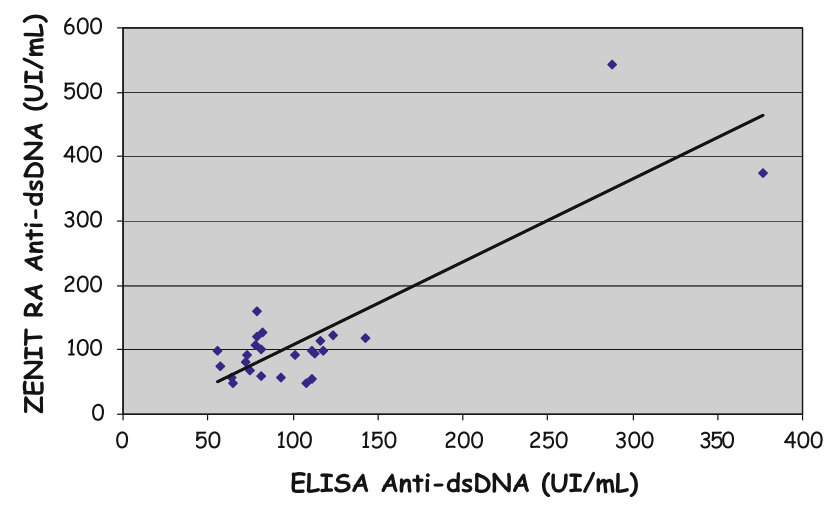

Fig. 2 Correlation between CLIA (Zenit RA) and ELISA for antidsDNA antibody determination $(r=0.85)$

Part III: daily use of Zenit RA for anti-ENA, anti ds-DNA and anti MPO or PR3 antibodies determinations

In this section, we studied various work modalities of the Zenit RA analyzer as batch mode, random access and stat priority. Required tests were performed every day after immunofluorescence screening in batch mode for the first patients, then, during the day in random or stat access.

So, 393 serum samples were analyzed: 259 for antidsDNA, 282 for anti-ENA, 54 for anti-MPO and 58 for anti-PR3 antibodies detection. A good agreement was found for each test, respectively, $94 \%$ for anti-dsDNA $(\kappa=0.75), 97 \%$ for anti-ENA $(\kappa=0.90), 98 \%$ for antiMPO $(\kappa=0.94)$ and $95 \%$ for anti-PR3 $(\kappa=0.86)$ antibody determination, despite the different nature of coated antigens on Zenit microparticles versus microplates.

Concerning anti-dsDNA antibodies, both tests used recombinant DNA as coated antigen and discrepant serum sample exhibited values near to cut off value of one out of the two tests. Figure 2 showed the good correlation
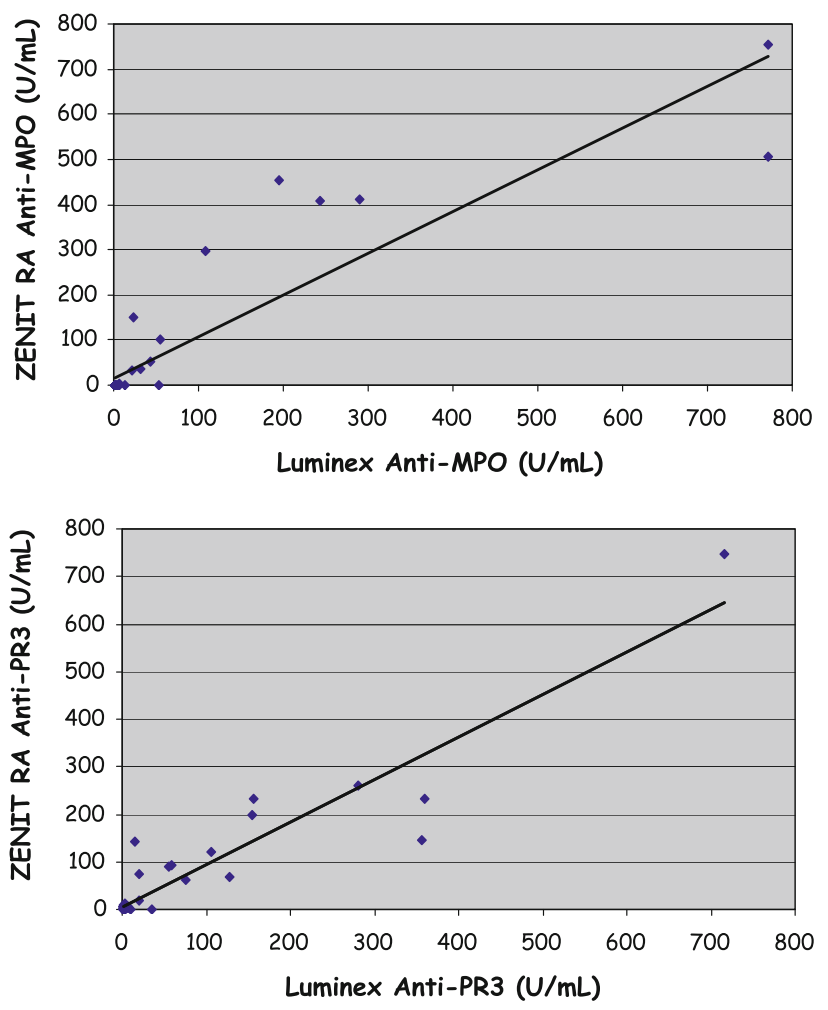

Fig. 3 Correlation between CLIA (Zenit RA) and Luminex for antiMPO $(r=0.91)$ and anti-PR3 $(r=0.94)$ antibody determination

$(r=0.85)$ between anti-dsDNA titers using CLIA (Zenit RA) versus ELISA. Furthermore, the cut off values were approximately similar in both tests.

Using Zenit RA anti-ENA screen test, two patients were not detected, whereas they had anti-Ro52 antibodies. As previously reported [11], hidden reactivities could exist when using blended Ro52 and Ro60 antigens, because they can mask each other's reactivity. 
In Fig. 3, we reported correlation curves of results obtained using CLIA and Luminex technology for the detection of anti-MPO $(r=0.91)$ and anti-PR3 $(r=0.94)$ antibodies, using both purified proteins as antigens and approximately similar cut off values.

These tests should easily substitute routine actual tests. Results were obtained in a short time. Indeed, the first result was available within $25 \mathrm{~min}$ and a batch of 30-40 samples required less than $1.5 \mathrm{~h}$ for one or two parameters per sample. No calibration curve was needed in each batch, only two controls were necessary to validate the run. In contrast, ELISA or Luminex tests required at least $2 \mathrm{~h}$ and several standard points were necessary for the calibration curve. So, they were actually performed in batch and not every day to optimize time and cost.

\section{Discussion}

Recent advances in diagnostic technologies enhanced the importance of antibody determination in autoimmune diseases, especially in early stage [12]. The practical approach in an autoimmunity laboratory tended to perform multi-parametric tests in a short time [13]. Automatization can improve the reproducibility and reduce interlaboratory variation, still a major problem in the analysis of autoantibodies. With this aim, CLIA have been developed by various manufacturers as DiaSorin with LIAISON ${ }^{\circledR}$ analyzer [14], Instrumentation Laboratory with BIO-FLASH ${ }^{\circledR}$ instrument [15] and Menarini Diagnostics with Zenit RA analyzer [3]. Reagents available on Zenit analyzer exhibit good diagnostic performances, regarding sensitivity, specificity, positive and negative predictive values. We agree with the recent study reported by Persijn [3], about a new set of automated CLIAs.

Observed discrepancies with our conventional routine tests were not higher than the discrepancies between other ELISA or other technologies, depending on various natures of antigens and lack of standardization [1].

The main Zenit's advantages were the complete automation and the flexibility of work modalities. The quality and security are provided by barcode reading for all reagents and samples, permitting full traceability of samples, reagents and operators. Different cartridges of reagents can stay on board in a refrigerated area with stability during 8 weeks and each calibration is stable for 2-3 weeks. Then, the analyzer is always ready to use after a daily automatic wake up. All types of tubes were accepted in the loading tray to limit decantation of serum samples and risk of mistake.

\section{Conclusion}

Zenit RA analyzer is easy to rapidly detect the most common autoantibodies in autoimmune diseases using CLIA. CLIA seems to be an attractive alternative to
ELISA, reduces labor as well as assay time. Zenit RA system has a potential to provide clinically useful data within a short time and is well adapted to determine antigenic specificities in daily practice and even in emergency.

Acknowledgments We thank the autoimmunity team from Menarini Diagnostics and particularly Sara Mattei and Frédérique M'Boro for support with the tests on Zenit RA and all the physicians who contributed to this study.

Conflict of interest None.

\section{References}

1. Conrad K, Roggenbuck D, Reinhold D, Sack U (2011) Autoantibody diagnostics in clinical practice. Autoimmun Rev. doi: 10.1016/j.autrev.2011.05.014

2. Wiik A, Cervera R, Haass M, Kallenberg C, Khamashta M, Peroni PL et al (2006) European attempts to set guidelines for improving diagnostics of autoimmune rheumatic disorders. Lupus 15:391-396

3. Persijn L, Decavele AS, Schouwers S, Devreese K (2011) Evaluation of a new set of automated chemiluminescense assays for anticardiolipin and anti-beta2-glycoprotein I antibodies in the laboratory diagnosis of the antiphospholipid syndrome. Thromb Res. doi:101016/j.thromres.2011.04.004

4. Aletaha D, Neogi T, Silman AJ, Funovits J et al (2010) 2010 Rheumatoid arthritis classification criteria: an American College of Rheumatology/European League against Rheumatism collaborative initiative. Ann Rheum Dis 69:1580-1588

5. Miyakis S, Lockshin MD, Atsumi T et al (2006) International consensus statement on an update of the classification criteria for definite antiphospholipid syndrome (APS). Thromb Haemost 4:295-306

6. Van Venrooij WJ, van Beers JJ, Pruijn GJ (2008) Anti-CCP antibody, a marker for the early detection of rheumatoid arthritis. Ann N Y Acad Sci 1143:268-285

7. Aggarval R, Liao K, Nair R et al (2009) Anti-citrullinated peptide antibody assays and their role in the diagnosis of rheumatoid arthritis. Arthritis Rheum 61:1472-1483

8. Arbuckle MR, McClain MT, Rubertone MB, Scofield RH, Dennis GJ, James JA et al (2003) Development of autoantibodies before the clinical onset of systemic lupus erythematosus. N Engl J Med 349:1526-1533

9. Tincani A, Filippini M, Scarsi M et al (2009) European attempts for the standardisation of the antiphospholipid antibodies. Lupus 10:913-919

10. De Laat B, De Groot PG (2011) Autoantibodies directed against domain I of beta2-glycoprotein 1. Curr Rheumatol Rep 13:70-76

11. Defendenti C, Atzeni F, Spina MF, Grosso S, Cereda A, Guercilena G, Bollani S, Saibene S, Puttini PS (2011) Clinical and laboratory aspects of Ro/SSA-52 autoantibodies. Autoimmun Rev 10:150-154

12. Tozzoli R (2007) Recent advances in diagnostic technologies and their impact in autoimmune diseases. Autoimmun Rev 6:334-340

13. Bossuyt X (2009) Clinical characteristics of a laboratory test: a practical approach in the autoimmune laboratory. Autoimmun Rev 9:431-435

14. Ghillani P, Rouquette AM, Desgruelles C et al (2007) Evaluation of the LIAISON ANA Screen assay for antibody testing in autoimmune diseases. Ann N Y Acad Sci 1109:407-413

15. Mahler M, Radice A, Sinico R et al (2011) Performance evaluation of a novel chemiluminescence assay for detection of antiGBM antibodies; an international multicenter study. Nephrol Dial Transpl. doi:10.1093/ndt/gfr203 